New nol ecul ar di agnost i c kit to assess Y-chromosome del et i ons in the Japanese popul at i on

\begin{tabular}{|c|c|}
\hline 著者 & 飯島 将司 \\
\hline 著者別表示 & I i j i ma Nasashi \\
\hline $\begin{array}{l}\text { j our nal or } \\
\text { publ i cat i on title }\end{array}$ & 博士論文本文Ful I \\
\hline 学位授与番号 & 13301甲第4102号 \\
\hline 学位名 & 博士 (医学) \\
\hline 学位授与年月日 & $201409-26$ \\
\hline URL & ht t p: //hdl . handl e. net /2297/41982 \\
\hline
\end{tabular}




\title{
New molecular diagnostic kit to assess Y-chromosome deletions in the Japanese population
}

\author{
Masashi Iijima, ${ }^{1}$ Eitetsu Koh, ${ }^{1}$ Kouji Izumi, ${ }^{1}$ Masaki Taya, ${ }^{1}$ Yuji Maeda, ${ }^{1}$ Kouichi Kyono, ${ }^{2}$ \\ Atsumi Yoshida ${ }^{3}$ and Mikio Namiki ${ }^{1}$ \\ ${ }^{1}$ Departments of Integrative Cancer Therapy and Urology, Kanazawa University Graduate School of Medical Science, Kanazawa, \\ Ishikawa, ${ }^{2}$ Kyono ART Clinic, Sendai, Miyagi, and ${ }^{3}$ Reproduction Center, Kiba Park Clinic, Tokyo, Japan
}

Abbreviations \& Acronyms
AZF = azoospermia factor
DNA = deoxyribonucleic
acid
EAA = European Academy
of Andrology
EMQN = European
Molecular Genetics Quality
Network
gr = green, red
sub-amplicon
P = palindrome
PCR = polymerase chain
reaction
SRY = sex-determining
region Y
STS = sequence-tagged site
TESE = testicular sperm
extraction
UCSC = University of
California, Santa Cruz
Ym = Y chromosomal
microdeletions
Yp = short arm of the Y
chromosome
Yq = long arm of the Y
chromosome

Correspondence: Eitetsu Koh M.D., Ph.D., Departments of Integrative Cancer Therapy and Urology, Kanazawa University Graduate School of Medical Science, 13-1 Takara-machi, Kanazawa, Ishikawa 920-8640, Japan. Email:

kohei@med.kanazawa-u.ac.jp

Received 4 January 2014; accepted 19 March 2014. Online publication 29 April 2014
Objectives: Deletions in the azoospermia factor regions are the most common known molecular genetic cause of human male infertility involving spermatogenetic failure. Testing for these deletions in Japanese DNA samples using conventional sequence-tagged site probes occasionally lead to considerable non-specific or faint products in the Japanese population. The aim of the present study was to evaluate the sensitivity and specificity of a newly developed kit for the detection of azoospermia factor microdeletions in the Japanese population.

Methods: Sequence-tagged site probes were reselected and the Luminex suspension array assay was carried out. Validation was retrospectively carried out with 2014 DNA sequences with known microdeletions, which were divided into four categories.

Results: Category 1 deletions that corresponded to the conventional classification of azoospermia factor deletion were present in 83 men (4.2\%), which can result in intrachromosomal homologous recombination. Kit data confirmed the presence of deletions of this type in DNA sequences known to harbor the azoospermia factor deletions. Category 2 deletions involved cytogenetic abnormalities in 28 men (1.4\%), whereas category 3 deletions in 759 men $(37.7 \%)$ were atypical classifications including the gr/gr deletion. As these deletions are thought to be a result of palindromic units and non-homologous recombination, these microdeletions might impact in the interpretation of some clinical findings. The rest of the 1145 cases (56.8\%) were assigned to category 4 as normal variants (polymorphism/no deletion).

Conclusions: The present findings show that this new kit offers good sensitivity and specificity with the advantage of saving in terms of cost and time.

Key words: azoospermia factor, detection kit, male infertility, microdeletion, Y chromosome.

\section{Introduction}

Male factors leading to infertility could account for up to $40-50 \%$ of total infertile couples. ${ }^{1}$ The origin of male-factor infertility remains largely unexplained. In healthy males, infertility could have a number of unknown causes, including genetic disorders. Approximately $7 \%$ of infertile men harbor microdeletions of the $\mathrm{Y}$ chromosome that are not detectable with routine karyotype analyses. ${ }^{2}$ Cytogenetic studies in infertile men have revealed a gene that controls spermatogenesis; this AZF is located on the Yq. ${ }^{3}$ Three spermatogenesis loci in Yq11 have been classified into three regions: $\mathrm{AZFa}, \mathrm{AZFb}$ and $\mathrm{AZFc}{ }^{4}$

The euchromatic region of the $\mathrm{Y}$ chromosome is currently characterized by three structurally distinct features, the $\mathrm{X}$-degenerated, $\mathrm{X}$-transposed and ampliconic sequences. The ampliconic region includes eight palindromes with pairs of duplicated amplicons in which the DNA sequences share more than $99.9 \%$ homology. ${ }^{5}$ These amplicons and sub-amplicons can serve as substrates for structural genomic rearrangement. Thus, AZF deletions can result from intrachromosomal homologous recombination events between non-reciprocal homologous sequences. ${ }^{6}$

From the clinical point of view, deletions in the AZF regions are the most common known molecular genetic cause of human male infertility involving spermatogenetic failure. ${ }^{7}$ Thus, the molecular diagnosis of Y-chromosomal microdeletions should be routinely carried out worldwide in the work-up of male infertility in men with azoospermia or severe oligozoospermia. The importance of a molecular genetics approach that includes the evaluation of AZF deletions must 
Fig. 1 Location of STS probes for control regions and for the AZFa region for use with the GENOSEARCH ${ }^{\text {TM }}$ AZF Deletion kit. Red, pseudoautosomal region; light green, euchromatin; blue, the heterochromatic region of the $Y$ chromosome. Probes SRY and sY3118 were Yp controls. Probe sY1251 was a control for the Yq centromere, and probe sY3159 was a control for the Yq euchromatin.

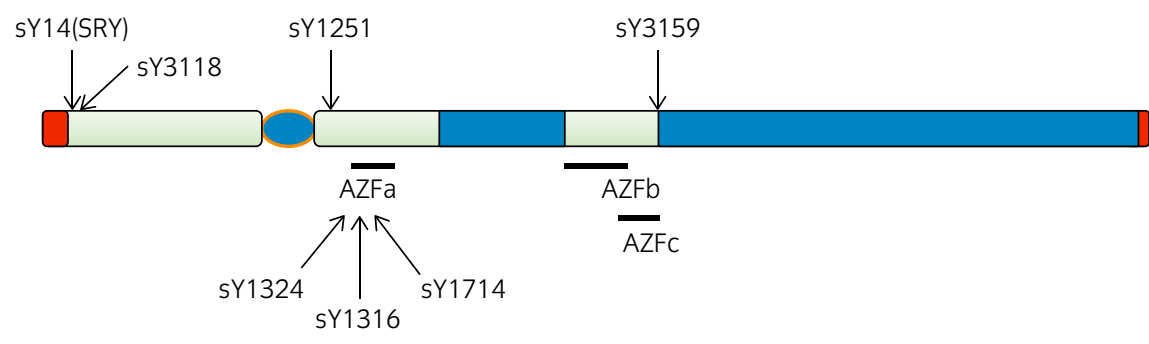

be emphasized for men considering assisted reproductive techniques including TESE, because this genetic defect is transmitted to their sons, affecting their fertility. ${ }^{8}$ Furthermore, this information is useful for the avoidance of unnecessary surgical therapy.

Nowadays, the gold standard for the diagnosis of microdeletions in the $\mathrm{AZFa}, \mathrm{AZFb}$ and $\mathrm{AZFc}$ regions utilizes PCR primers, including primers sY84, sY86, sY127, sY134, sY254 and sY255. ${ }^{9}$ When we previously tested Japanese patients using these eight probes, considerable non-specific or faint bands were occasionally observed in the PCR products, particularly after the use of the sY254 and sY255 probes in the $\mathrm{AZFc}$ region. Re-evaluation frequently necessitated the use of several adjacent STS markers. ${ }^{10}$

To improve the sensitivity and specificity of the detection of microdeletions in the $\mathrm{Y}$ chromosome, we developed a new kit for the detection of molecular Y-chromosome deletions by re-selecting STS probes and carrying out multiplex target detection on the Luminex suspension array platform (Luminex, Austin, TX, USA). Here, we report the results of a retrospective evaluation of the new molecular Y-chromosome deletion kit using DNA with previously determined genetic phenotypes.

\section{Methods}

\section{DNA sources and samples}

The present study was approved by the ethics committee of the Kanazawa University Graduate School of Medical Science. All participants had granted informed consent for a previous study. Genomic DNA was sampled in a male infertility clinic, and stored after diagnosing the presence or absence of Y-chromosomal microdeletions between 1999 April and 2012 December. This genetic diagnosis included the identification of AZF deletions. The samples were anonymized and made available for research after approval by the appropriate ethics committee.

We retrospectively analyzed genomic DNA samples from 2014 anonymized subjects who visited the clinic with male infertility as their chief complaint. Deletions in these DNA samples were confirmed using in-house detection methods. DNA samples from patients with Klinefelter syndrome or hypogonadotrophic hypogonadism were not included in the present investigation.

\section{In-house detection probes}

All DNA samples were screened for Y-chromosomal microdeletions according to the guidelines of the EAA and the EMQN. These guidelines recommend the following first-choice STS primers: two STS in AZFa (sY86 and sY84), two in AZFb
(sY127 and sY134) and two in AZFc (sY254 and sY255). ${ }^{9}$ These STS probes previously yielded reproducible results.

All DNA samples were evaluated for deletions using the following in-house STS probes: for AZFa, probes sY82, sY84 and sY86; for AZFb, probes sY1264, sY1235, sY1227, sY1228, sY117, sY280, sY127, sY134, sY135, sY258, sY142 and sY143; for AZFc, probes sY1161, sY1191, sY1197, sY1291, sY1125, sY1054, sY1206, sY1201, sY255 and sY254. ${ }^{11}$

These probes were designed according to EAA/EMQN guidelines, and produced with the UCSC Genome Bioinformatics and the GenBank database. The human reference sequence (UCSC version hg16, 17 and 18) was based on National Center for Biotechnology Information Build 34 (July 2003), 35 (May 2004) and 36 (March 2006) produced by the International Human Genome Sequencing Consortium (http:// genome.ucsc.edu/index.html).

All DNA samples were subjected to STS testing to confirm the presence or absence of deletions.

\section{STS probes in the GENOSEARCH ${ }^{\mathrm{TM}}$ AZF Deletion kit}

STS probes for the $\mathrm{Y}$ chromosome were chosen as markers of either single or double sequence copies using MSY Breakpoint Mapper (http://breakpointmapper.wi.mit.edu/) and UniSTS (http://www.ncbi.nlm.nih.gov/unists). A total of 20 STS probes were located on the Y chromosome. Probe sY757 (SOX3) served as a control probe for the $\mathrm{X}$ chromosome. The controls for the Yp were sY14 (SRY) and sY3118, and the controls for Yq were sY1251 and sY3159. Probes sY1251 and sY3159 were controls for the proximal and distal Y chromosome, respectively. Probes sY1324, sY1316 and sY1714 were used for the detection of AZFa deletions (Fig. 1). Figure 2 shows the locations (proximal to distal) of the following probes in the $\mathrm{AZFb}$ and AZFc regions: sY1024, sY1967, sY1309, sY3199, sY1233, sY3010, sY2990, sY1197, sY1191, sY1307, sY1291, sY2858 and sY1206. All of these probes are single-copy probes, with the exceptions of sY1967, sY1307, sY2858 and sY1206, which are present in two copies on the $\mathrm{Y}$ chromosome.

\section{Multiplex PCR}

Genomic DNA was prepared from patient peripheral blood lymphocytes with several commercial extraction kits according to the manufacturers' protocols. PCR was carried out in $25 \mu \mathrm{L}$ of PCR buffer $(20 \mathrm{mmol} / \mathrm{L}$ Tris- $\mathrm{HCl}$ [pH 8.3], $30 \mathrm{mmol} / \mathrm{L}$ $\mathrm{KCl}, 2.5 \mathrm{mmol} / \mathrm{L} \mathrm{MgCl2}$ ) containing $200 \mu \mathrm{mol} / \mathrm{L}$ of each deoxyribonucleoside triphosphate, with 0.625 U TaqDNA Polymerase (Roche Diagnostics, Mannheim, Germany), 50-100 ng 


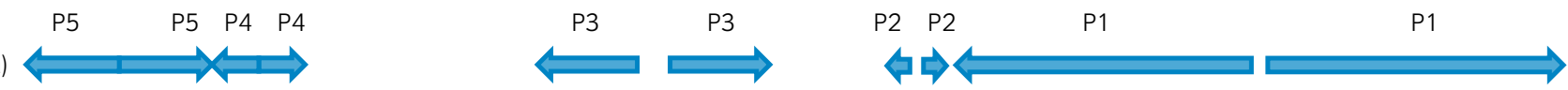

(b)
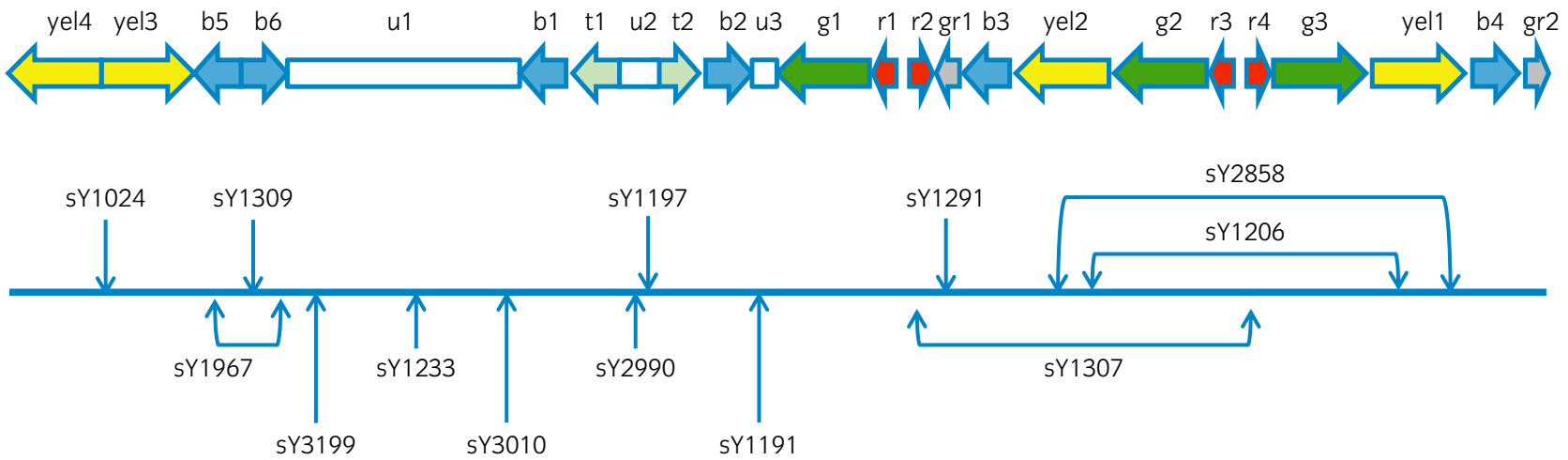

(d)

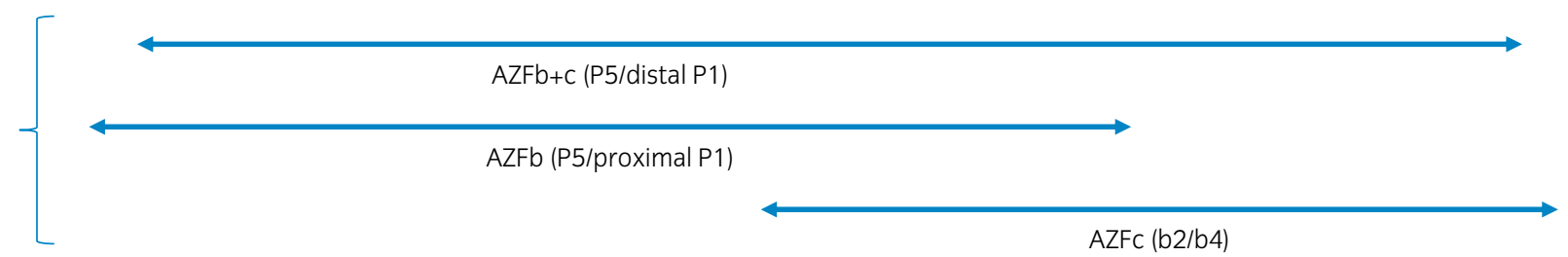

Fig. 2 AZFb and AZFC regions in the Y chromosome. (a) P structure in the Y chromosome. Rightward-facing arrows denote the 3' to 5' direction. (b) Sub-amplicon structure of the AZFc region. Identical pairs of amplicons appear in the same color. b, Blue sub-amplicon; g, green sub-amplicon; gr, grey sub-amplicon; $r$, red sub-amplicon; t, tan sub-amplicon; yel, yellow sub-amplicon. (c) Locations of the probes used with the GENOSEARCH ${ }^{\mathrm{TM}}$ AZF Deletion kit. (d) The AZFb and AZFc regions. Microdeletions in AZFb and AZFC result from non-reciprocal intrachromosomal recombination events between homologous sequences.

of genomic DNA and each primer at a final concentration of $0.2 \mu \mathrm{mol} / \mathrm{L}$. All PCR primers were biotinylated. The PCR profile consisted of one cycle of 2 min at $93^{\circ} \mathrm{C}$, followed by 45 cycles of $1 \mathrm{~min}$ at $93^{\circ} \mathrm{C}, 30 \mathrm{~s}$ at the annealing temperature of $59^{\circ} \mathrm{C}$ and $60 \mathrm{~s}$ at $70^{\circ} \mathrm{C}$, with a final cycle of $5 \mathrm{~min}$ at $70^{\circ} \mathrm{C}$. Amplifications were carried out on a GeneAmp 9700 thermal cycler (Applied Biosystems, Foster City, CA, USA).

\section{Luminex XMAP technology}

Results from the GENOSEARCH ${ }^{\mathrm{TM}}$ AZF Deletion kit (MBL, Nagoya, Japan) of development stage were confirmed by Luminex xMAP technology. To detect deletions in the AZF regions, amplification by sequence-specific probes and hybridization of each solid phase-binding STS were carried out. The probes were 15-30mers that encompassed the sequence in the amplification region for each set of biotinylated primers. PCR products were mixed with the microbeads; the probes bound these beads, enabling multiple hybridization reactions in the same tube.

Each $30-\mu \mathrm{L}$ hybridization reaction consisted of $5 \mu \mathrm{L}$ PCR product, $20 \mu \mathrm{L} 1 \times$ hybridization buffer $(75 \mathrm{mmol} / \mathrm{L}$ Tris- $\mathrm{HCl}$ [pH 8.0], $0.6 \mathrm{mmol} / \mathrm{L}$ ethylenediaminetetraacetic acid [pH 8.0], $0.15 \%$ surfactant, $4.5 \mathrm{~mol} / \mathrm{L}$ tetramethylammonium chloride) and $5 \mu \mathrm{L}$ probe-binding beads. The hybridization reactions were carried out at $95^{\circ} \mathrm{C}$ for $2 \mathrm{~min}$ and $52^{\circ} \mathrm{C}$ for $30 \mathrm{~min}$. After the incubation, the beads were washed twice with $70 \mu \mathrm{L}$ of phosphate-buffered saline-Tween with centrifugation for $1 \mathrm{~min}$ at $2000 \mathrm{~g}$.
After washing, the samples were labeled with streptavidinphycoerythrin and incubated at $52^{\circ} \mathrm{C}$ for $15 \mathrm{~min}$. This hybridization between the PCR-amplified product and the probes on the fluorescently labeled beads allowed the quantification of the number of probes corresponding to each STS marker after flow cytometry based on the Luminex system. Each measurement encompassed at least 50 beads; the median phycoerythrin intensity was considered.

To determine the presence of deletions, we set individual cut-off values for the fluorescence intensity of each bead-bound probe; if the intensity was below the cut-off, the sequence was defined as harboring a deletion. Samples from healthy female subjects and a negative control of distilled water were used to determine the individual cut-offs.

\section{Validation}

We tested 2014 samples with our in-house kit. Our laboratory carried out validation with external control DNA samples dispatched from the EMQN every year.

\section{Results}

\section{General results and probe characteristics}

The current investigation evaluated 2014 DNA samples. The mean participant age was 35.0 \pm 7.0 years (range 19-72 years). All DNA samples that had previously been determined to harbor deletions associated with a chief complaint of male infertility were analyzed. The deletions were classified into 


\begin{tabular}{|c|c|c|c|c|c|c|c|c|c|c|c|c|c|c|c|c|c|c|c|c|c|c|c|c|c|c|c|}
\hline & & & & & & & & & & & & & & & & & & & & & & & $\downarrow$ & $\downarrow$ & & & \\
\hline & & - & $\sim$ & $m$ & $\nabla$ & เ & 0 & $\wedge$ & $\infty$ & $a$ & & $a$ & $\mp$ & $\stackrel{\sim}{-}$ & $\stackrel{m}{\square}$ & 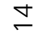 & $\stackrel{\llcorner}{\leftarrow}$ & $\stackrel{0}{-}$ & $\approx$ & $\stackrel{\infty}{-}$ & $\stackrel{2}{-}$ & 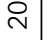 & 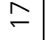 & $\stackrel{\sim}{\sim}$ & $\stackrel{9}{-1}$ & $\bar{\sim}$ & \\
\hline Pattern & $\begin{array}{c}\text { Sample } \\
\text { no. }\end{array}$ & \begin{tabular}{l}
$\hat{n}$ \\
\multirow{n}{n}{}
\end{tabular} & $\frac{\nabla}{i}$ & $\frac{\infty}{\frac{m}{m}}$ & $\frac{i n}{\stackrel{i n}{\Sigma}}$ & $\underset{i}{\stackrel{D}{m}}$ & $\frac{0}{\frac{m}{m}}$ & $\frac{\frac{\nabla}{\pi}}{\frac{\pi}{n}}$ & 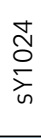 & $\begin{array}{l}0 \\
o \\
\frac{i}{i}\end{array}$ & $\begin{array}{l}\frac{a}{0} \\
\frac{m}{i}\end{array}$ & $\begin{array}{l}\hat{0} \\
\frac{1}{i} \\
i\end{array}$ & $\frac{\sigma}{\frac{\sigma}{m}} \frac{}{\frac{m}{\omega}}$ & 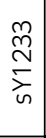 & $\begin{array}{l}0 \\
\frac{0}{n} \\
\frac{m}{n}\end{array}$ & $\begin{array}{l}8 \\
\circ \\
\frac{1}{2} \\
\text { in }\end{array}$ & $\frac{\hat{\sigma}}{\frac{1}{i}}$ & $\begin{array}{l}\bar{\sigma} \\
\frac{\bar{z}}{n}\end{array}$ & $\begin{array}{l}\frac{D}{m} \\
\frac{m}{i}\end{array}$ & $\frac{\bar{a}}{\stackrel{i}{i}}$ & $\begin{array}{l}\infty \\
\infty \\
\infty \\
i \\
i \\
i\end{array}$ & $\begin{array}{l}\stackrel{D}{i} \\
\frac{i}{i}\end{array}$ & $\frac{\hat{D}}{\frac{m}{i}}$ & $\frac{\stackrel{0}{0}}{\frac{i}{i}}$ & $\begin{array}{l}\infty \\
\stackrel{\infty}{\infty} \\
\stackrel{\sim}{i} \\
i\end{array}$ & 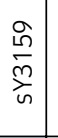 & Deletions \\
\hline 4 & 5 & 1 & 1 & 1 & 1 & 0 & 0 & 0 & 1 & 1 & 1 & 1 & 1 & 1 & 1 & 1 & 1 & 1 & 1 & 0 & 1 & 1 & 1 & 1 & 1 & 11 & AZFa \\
\hline 5 & 2 & 1 & 1 & 1 & 1 & 0 & 0 & 0 & 1 & 1 & 1 & 1 & 1 & 1 & 1 & 1 & 1 & 1 & 1 & 1 & 1 & 1 & 1 & 1 & 1 & \begin{tabular}{l|l}
1 & 1 \\
\end{tabular} & AZFa \\
\hline 7 & 2 & 1 & 1 & 1 & 1 & 1 & 1 & 1 & 0 & 0 & 0 & 0 & 0 & 0 & 0 & 0 & 0 & 0 & 0 & 0 & $1 *$ & 0 & 0 & 0 & 1 & \begin{tabular}{l|l}
1 & 1 \\
\end{tabular} & AZFb+c (P5/distal P1) \\
\hline 8 & 1 & 1 & 1 & 1 & 1 & 1 & 1 & 1 & 0 & 0 & 0 & 0 & 0 & 0 & 0 & 0 & 0 & 0 & $1 *$ & 0 & 1 & 1 & 1 & 1 & 1 & \begin{tabular}{l|l}
1 & 1 \\
\end{tabular} & AZFb (P5/proximal P1) \\
\hline 14 & 10 & 1 & 1 & 1 & 1 & 1 & 1 & 1 & 1 & 0 & 0 & 0 & 0 & 0 & 0 & 0 & 0 & 0 & 0 & 0 & $1 *$ & 0 & 0 & 0 & 1 & \begin{tabular}{l|l}
1 & 1 \\
\end{tabular} & AZFb+C (P5/distal P1) \\
\hline 30 & 62 & 1 & 1 & 1 & 1 & 1 & 1 & 1 & 1 & 1 & 1 & 1 & 1 & 1 & 1 & 1 & 1 & 0 & 0 & 0 & 0 & 0 & 0 & 0 & 0 & \begin{tabular}{l|l}
1 & 1 \\
\end{tabular} & AZFc (b2/b4) \\
\hline 31 & 1 & 1 & 1 & 1 & 1 & 1 & 1 & 1 & 1 & 1 & 1 & 1 & 1 & 1 & 1 & 1 & 1 & 0 & 0 & 0 & $1 *$ & 0 & 0 & 0 & 1 & \begin{tabular}{l|l}
1 & 1 \\
\end{tabular} & AZFc (b2/b4) \\
\hline
\end{tabular}

Fig. 3 Classical AZF microdeletions (category 1). Within the body of the figure, "0" denotes "absence," "1" denotes "presence" and " $1 *$ " denotes duplicated copies of a DNA sequence. Blue arrows highlight data from the same probe. Possible deletions are colored light blue.

40 patterns according to differences in adjoining patterns (Fig. S1).

Here, we present broad genetic information about microdeletions of the $\mathrm{Y}$ chromosome that are pertinent for clinicians or researchers investigating Japanese populations. For convenience, we grouped the results from our kit into four categories. Probe sY1291 is especially pertinent for Japanese populations; this marker was previously used for the detection of both the $g r / g r$ (green-red sub-amplicon) deletion and $\mathrm{Y}$ haplogroup marker D. ${ }^{12,13}$ One-third of Japanese males carry the deletion probed by sY1291. ${ }^{14-16}$ Therefore, the deletion of the sequence probed by sY1291 would not be taken into account during the evaluation of all but $g r / g r$ deletions (pattern 39).

Furthermore, a pair of palindromes was duplicated in the set of DNA samples included in this investigation, yielding double hybridization of STS probes; for example, for probes SY1967, sY1307, sY2858 and sY1206. These probes might not always detect the deletion of the duplicated region when implemented in a PCR-based evaluation. When non-contiguous deletions were observed, such as in patterns $7,8,11,14,15,18,25,26$, $31,32,35$ and 36 , we avoided non-sequential regions when designating the deletion location (Fig. S1, 1*).

\section{Categorization of deletion patterns and number in a Japanese population}

We classified the detected deletion patterns into the following four categories: category 1, classical AZF microdeletions; category 2, Y chromosome long-arm terminal deletions, including heterochromatin deletion; category 3 , subclassification of Yq microdeletions; category 4, miscellaneous deletions (polymorphisms).

Category 1 deletions in 83 men (4.2\%), category 2 deletion in $28(1.4 \%)$ and category 3 deletion in $759(37.7 \%)$ including $g r / g r$ deletion were indicated. The remaining 1145 cases $(56.8 \%)$ were assigned to category 4 in the present study.

For category 1 deletions, we divided the conventional concept of the AZF region into regions $\mathrm{AZFa}, \mathrm{AZFb}$ (P5/ proximal $\mathrm{P} 1), \mathrm{AZFb}+\mathrm{c}(\mathrm{P} 5 /$ distal $\mathrm{P} 1$; recombination between palindrome5 and distal palindrome1) (Fig. 2) and AZFc (b2/ b4). The P5/distal P1 recombination eliminated the intervening homologous sequences. The "b2/b4" categorization captures a recombination event between sub-amplicons b2 and b4
(Fig. 2b,d). This deletion category is based on the EAA/EMQN guidelines. ${ }^{17}$ This conventional classification encompasses the probes indicated in Figure 2c, and yielded the corresponding patterns reported in Figure 3. There were seven, one, 12 and 63 samples that harbored deletions in $\mathrm{AZFa}, \mathrm{AZFb}, \mathrm{AZFb}+\mathrm{c}$, and AZFc, respectively (Fig. 3). All AZF deletions were confirmed by in-house STS probes. Therefore, the sensitivity and the specificity of our new kit were $100 \%$ for the detection of category-1 AZF deletions.

Category 1 deletions result in the recombination of the intervening homologous sequences between duplicated palindromes (Fig. 2d). Thus, the term "microdeletion" implies that a part of the deletion of the inner euchromatin region of $\mathrm{Yq}$ is a result of homologous recombination. We suggest differentiating microdeletion from partial Yq terminal deletion like following category 2 deletions.

With regard to category 2 deletions, probe sY3159 is located the end of the Yq euchromatin (Fig. 1). Deletion of this sequence could be accompanied by elimination of the distal $\mathrm{Y}$ chromosome end, including the heterochromatin. Patterns 3 (Yq I), 6 (Yq II), 13 (Yq III), 17 (Yq IV), 19 (Yq V) and 29 (Yq VI) were deleted from the distal Yq (Fig. 4). These deletions were also detected by evaluating karyotype analyses (Table S1). Yq terminal deletions I-VI appeared in one, four, six, five, three and three samples, respectively. Interestingly, all patients carrying pattern 2 were diagnosed as XX male.

According to deletions of sY3159, patterns 37 indicated a normal karyotype. One sample showed pattern 38, deletion of sY3159. This karyotype was 46, XY (Fig. 6). Deletion of sY3159 alone was classified as a polymorphism present in the Japanese population; therefore, pattern 25 was classified as Ym-8 (Fig. 5).

Category 3 included atypical Y microdeletions (Fig. 5). These deletions most likely involved palindromic units, unlike the intrachromosomal non-homologous recombination that underlies category-1 deletions. Although few category 3 deletions were detected (Fig. 5; Ym-1 to Ym-7, Ym-9, Ym-10 and Ym-13 deletions occurred in 2, 1, 2, 1, 1, 5, 1, 2, 1, and 2 samples, respectively), these deletions are of clinical significance, and are the focus of a separate investigation.

In contrast, patterns 25 and $26(\mathrm{Ym}-8)$, pattern 28 and 32 (Ym-11), and pattern 39 (Ym-12) indicate partial deletions of 


\begin{tabular}{|c|c|c|c|c|c|c|c|c|c|c|c|c|c|c|c|c|c|c|c|c|c|c|c|c|c|c|c|}
\hline & & & & & & & & & & & & & & & & & & & & & & 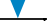 & & & & & \\
\hline & & - & $\sim$ & $m$ & $\nabla$ & เ & 0 & $n$ & $\infty$ & $a$ & $\stackrel{-}{\circ}$ & $a$ & 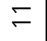 & $\simeq$ & $\stackrel{m}{\longrightarrow}$ & $\stackrel{\Xi}{\square}$ & $\stackrel{n}{\leftarrow}$ & $\stackrel{\circ}{\circ}$ & $£$ & $\stackrel{\infty}{-}$ & $\stackrel{a}{-}$ & 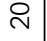 & 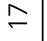 & $\stackrel{\sim}{2}$ & $\stackrel{a}{-}$ & $\bar{\sim}$ & \\
\hline Pattern & $\begin{array}{c}\text { Sample } \\
\text { no. }\end{array}$ & 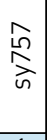 & $\frac{8}{i}$ & $\frac{\infty}{\frac{m}{m}}$ & $\frac{\stackrel{\sqrt[i n]{N}}{i}}{i n}$ & $\frac{\stackrel{d}{m}}{i}$ & $\frac{0}{\frac{m}{m}}$ & $\frac{\frac{\nabla}{\pi}}{\frac{\pi}{i}}$ & $\begin{array}{l}\stackrel{d}{0} \\
\frac{\delta}{i}\end{array}$ & $\begin{array}{l}\frac{1}{0} \\
\frac{1}{i} \\
\frac{1}{n}\end{array}$ & $\begin{array}{l}0 \\
\frac{0}{m} \\
\frac{\pi}{i}\end{array}$ & $\begin{array}{l}\hat{0} \\
\frac{2}{i} \\
\frac{1}{n}\end{array}$ & $\frac{2}{\frac{2}{m}} \frac{m}{\frac{m}{n}}$ & $\underset{⿱ 亠 䒑}{\stackrel{N}{i}}$ & $\frac{0}{\frac{0}{0}}$ & $\begin{array}{l}8 \\
\text { \&े } \\
\frac{N}{\omega}\end{array}$ & $\frac{\hat{\sigma}}{\frac{i}{i}}$ & $\frac{\bar{\sigma}}{\frac{\bar{z}}{\omega}}$ & $\begin{array}{l}\frac{\hat{o}}{m} \\
\frac{m}{i}\end{array}$ & $\begin{array}{c}\bar{\vdots} \\
\underset{i}{i}\end{array}$ & $\begin{array}{l}\stackrel{\infty}{\infty} \\
\underset{\sim}{\infty} \\
i \\
\text { in }\end{array}$ & $\underset{i}{\stackrel{D}{i}}$ & $\begin{array}{l}\frac{\hat{O}}{m} \\
\frac{m}{i}\end{array}$ & $\frac{\stackrel{D}{i}}{i}$ & $\begin{array}{l}\infty \\
\stackrel{\infty}{\infty} \\
\stackrel{\sim}{\sim} \\
i\end{array}$ & $\frac{a}{\frac{\omega}{m}} \frac{m_{n}^{m}}{m}$ & Deletions \\
\hline 1 & 2 & 1 & 0 & 0 & 0 & 0 & 0 & 0 & 0 & 0 & 0 & 0 & 0 & 0 & 0 & 0 & 0 & 0 & 0 & 0 & 0 & 0 & 0 & 0 & 0 & 0 & Whole Y \\
\hline 2 & 4 & 1 & 1 & 1 & 0 & 0 & 0 & 0 & 0 & 0 & 0 & 0 & 0 & 0 & 0 & 0 & 0 & 0 & 0 & 0 & 0 & 0 & 0 & 0 & 0 & 0 & Yp \\
\hline 3 & 1 & 1 & 1 & 1 & 1 & 0 & 0 & 0 & 0 & 0 & 0 & 0 & 0 & 0 & 0 & 0 & 0 & 0 & 0 & 0 & 0 & 0 & 0 & 0 & 0 & 0 & Yq I (distal to AZFa) \\
\hline 6 & 4 & 1 & 1 & 1 & 1 & 1 & 1 & 1 & 0 & 0 & 0 & 0 & 0 & 0 & 0 & 0 & 0 & 0 & 0 & 0 & 0 & 0 & 0 & 0 & 0 & 0 & Yq II (distal to AZFb) \\
\hline 13 & 6 & 1 & 1 & 1 & 1 & 1 & 1 & 1 & 1 & 0 & 0 & 0 & 0 & 0 & 0 & 0 & 0 & 0 & 0 & 0 & 0 & 0 & 0 & 0 & 0 & 0 & Yq III (distal to AZFb) \\
\hline 17 & 5 & 1 & 1 & 1 & 1 & 1 & 1 & 1 & 1 & 1 & 1 & 1 & 0 & 0 & 0 & 0 & 0 & 0 & 0 & 0 & 0 & 0 & 0 & 0 & 0 & 0 & Yq IV (distal to AZFb) \\
\hline 19 & 3 & 1 & 1 & 1 & 1 & 1 & 1 & 1 & 1 & 1 & 1 & 1 & 1 & 1 & 0 & 0 & 0 & 0 & 0 & 0 & 0 & 0 & 0 & 0 & 0 & 0 & Yq V (distal to AZFb) \\
\hline 29 & 3 & 1 & 1 & 1 & 1 & 1 & 1 & 1 & 1 & 1 & 1 & 1 & 1 & 1 & 1 & 1 & 1 & 0 & 0 & 0 & 0 & 0 & 0 & 0 & 0 & 0 & Yq VI (distal to AZFb) \\
\hline
\end{tabular}

Fig. 4 Y chromosome long-arm terminal deletions, including heterochromatin (category 2). Blue arrows highlight data from the same probe. Possible deletions are colored light blue.

\begin{tabular}{|c|c|c|c|c|c|c|c|c|c|c|c|c|c|c|c|c|c|c|c|c|c|c|c|c|c|c|c|}
\hline & & & & & & & & & & & & & & & & & & & & & $\downarrow$ & $v$ & $V$ & $\downarrow$ & $\downarrow$ & & \\
\hline & & - & N & $m$ & $\nabla$ & 几 & 0 & $\wedge$ & $\infty$ & $a$ & & $a$ & $=$ & $\simeq$ & $\stackrel{m}{-}$ & $\stackrel{\nabla}{\sim}$ & $\stackrel{2}{\leftarrow}$ & $\stackrel{0}{\sim}$ & 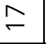 & $\stackrel{\infty}{-}$ & $\stackrel{2}{\square}$ & $\stackrel{\sim}{\circ}$ & $\approx$ & $\stackrel{\sim}{2}$ & $\stackrel{q}{-}$ & $\bar{\sim}$ & \\
\hline Pattern & $\begin{array}{c}\text { Sample } \\
\text { no. }\end{array}$ & 望 & $\sum_{i}^{\frac{\pi}{n}}$ & $\frac{\infty}{\frac{\infty}{m}}$ & 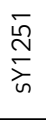 & $\underset{⿱ 亠}{\stackrel{d}{m}}$ & $\frac{0}{\frac{m}{i}}$ & $\frac{\frac{D}{\pi}}{\frac{\pi}{i}}$ & $\frac{\stackrel{d}{d}}{\frac{\delta}{i}}$ & $\begin{array}{l}\hat{\sigma} \\
\frac{1}{i} \\
\frac{1}{n}\end{array}$ & $\frac{\partial}{m_{i}^{m}}$ & $\begin{array}{l}\frac{2}{2} \\
\frac{i}{i}\end{array}$ & $\frac{2}{\frac{\sigma}{m}}$ & 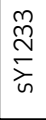 & $\begin{array}{l}\frac{0}{\circ} \\
\frac{m}{i n}\end{array}$ & $\begin{array}{l}8 \\
\vdots \\
\frac{N}{n}\end{array}$ & & $\frac{\bar{a}}{\frac{\pi}{i}}$ & $\frac{\hat{m}}{\sum_{i}^{m}}$ & 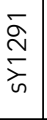 & $\begin{array}{l}\omega_{0}^{\infty} \\
\underset{\sim}{\sim} \\
i n\end{array}$ & $\frac{\substack{0 \\
\frac{1}{i}}}{\text { in }}$ & $\frac{\hat{m}}{\sum_{i}^{m}}$ & $\frac{\stackrel{D}{N}}{i=}$ & 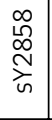 & $\frac{\frac{a}{m}}{\frac{m}{m}}$ & Deletions \\
\hline 9 & 1 & 1 & 1 & 1 & 1 & 1 & 1 & 1 & 0 & 0 & 0 & 0 & 0 & 1 & 1 & 1 & 1 & 1 & 1 & 1 & 1 & 1 & 1 & 1 & 1 & 1 & $\mathrm{Ym}-2$ (P5+P4) \\
\hline 10 & 1 & 1 & 1 & 1 & 1 & 1 & 1 & 1 & 0 & 0 & 0 & 0 & 1 & 1 & 1 & 1 & 1 & 1 & 1 & 0 & 1 & 1 & 1 & 1 & 1 & 1 & $\mathrm{Ym}-1$ (P5+P4) \\
\hline 11 & 1 & 1 & 1 & 1 & 1 & 1 & 1 & 1 & 0 & $1 *$ & 0 & 1 & 1 & 1 & 1 & 0 & 1 & 1 & 1 & 1 & 1 & 1 & 1 & 1 & 1 & 1 & Ym-1 (P5+P4) \\
\hline 15 & 1 & 1 & 1 & 1 & 1 & 1 & 1 & 1 & 1 & 0 & 0 & 0 & 0 & 0 & 0 & 0 & 0 & 0 & $1 *$ & 0 & 1 & 1 & 1 & 1 & 1 & 1 & Ym-3 (AZFb partial) \\
\hline 16 & 1 & 1 & 1 & 1 & 1 & 1 & 1 & 1 & 1 & 0 & 0 & 0 & 0 & 0 & 0 & 0 & 0 & 0 & 1 & 1 & 1 & 1 & 1 & 1 & 1 & 1 & Ym-3 (AZFb partial) \\
\hline 18 & 1 & 1 & 1 & 1 & 1 & 1 & 1 & 1 & 1 & 1 & 1 & 1 & 0 & 0 & 0 & 0 & 0 & 0 & $1 *$ & 0 & 1 & 1 & 1 & 1 & 1 & 1 & Ym-4 (AZFb partial) \\
\hline 20 & 5 & 1 & 1 & 1 & 1 & 1 & 1 & 1 & 1 & 1 & 1 & 1 & 1 & 1 & 0 & 0 & 0 & 0 & 0 & 0 & 0 & 0 & 0 & 0 & 0 & 1 & Ym-6 (P3+P2+P1) \\
\hline 21 & 1 & 1 & 1 & 1 & 1 & 1 & 1 & 1 & 1 & 1 & 1 & 1 & 1 & 1 & 0 & 0 & 0 & 1 & 1 & 1 & 1 & 1 & 1 & 1 & 1 & 1 & Ym-5 (P3) \\
\hline 24 & 1 & 1 & 1 & 1 & 1 & 1 & 1 & 1 & 1 & 1 & 1 & 1 & 1 & 1 & 1 & 0 & 0 & 0 & 0 & 0 & 0 & 0 & 0 & 0 & 0 & 1 & $\mathrm{Ym}-7$ (P3+P2+P1) \\
\hline 25 & 1 & 1 & 1 & 1 & 1 & 1 & 1 & 1 & 1 & 1 & 1 & 1 & 1 & 1 & 1 & 0 & 0 & 0 & $1 *$ & 0 & 1 & 1 & 1 & 1 & 1 & 0 & Ym-8 (b1/b3) \\
\hline 26 & 17 & 1 & 1 & 1 & 1 & 1 & 1 & 1 & 1 & 1 & 1 & 1 & 1 & 1 & 1 & 0 & 0 & 0 & $1 *$ & 0 & 1 & 1 & 1 & 1 & 1 & 1 & Ym-8 (b1/b3) \\
\hline 27 & 2 & 1 & 1 & 1 & 1 & 1 & 1 & 1 & 1 & 1 & 1 & 1 & 1 & 1 & 1 & 0 & 0 & 0 & 1 & 1 & 1 & 1 & 1 & 1 & 1 & 1 & Ym-9 (P3) \\
\hline 28 & 1 & 1 & 1 & 1 & 1 & 1 & 1 & 1 & 1 & 1 & 1 & 1 & 1 & 1 & 1 & 0 & 0 & 1 & 1 & 0 & 1 & 1 & 1 & 1 & 1 & 1 & Ym-10 (P3) \\
\hline 32 & 1 & 1 & 1 & 1 & 1 & 1 & 1 & 1 & 1 & 1 & 1 & 1 & 1 & 1 & 1 & 1 & 1 & 0 & $1 *$ & 0 & 1 & 1 & 1 & 1 & 1 & 1 & Ym-11 (b2/b3) \\
\hline 33 & 32 & 1 & 1 & 1 & 1 & 1 & 1 & 1 & 1 & 1 & 1 & 1 & 1 & 1 & 1 & 1 & 1 & 0 & 1 & 1 & 1 & 1 & 1 & 1 & 1 & 1 & $\mathrm{Ym}-11$ (b2/b3) \\
\hline 34 & 2 & 1 & 1 & 1 & 1 & 1 & 1 & 1 & 1 & 1 & 1 & 1 & 1 & 1 & 1 & 1 & 1 & 1 & 0 & 0 & 0 & 0 & 0 & 0 & 0 & 1 & Ym-13 (P1) \\
\hline 39 & 690 & 1 & 1 & 1 & 1 & 1 & 1 & 1 & 1 & 1 & 1 & 1 & 1 & 1 & 1 & 1 & 1 & 1 & 1 & 0 & 1 & 1 & 1 & 1 & 1 & 1 & Ym-12 (gr/gr) \\
\hline
\end{tabular}

Fig. 5 Y chromosome microdeletion (subclassification; category 3). Blue arrows highlight data from the same probe. Possible deletions are colored light blue.

AZFc as a result of homologous recombination between subamplicons b1/b3, b2/b3 and gr/gr, respectively (Fig. 5). These deletions have already been investigated. ${ }^{18-20}$ There were 18, 33 and 690 samples in the present study with Ym-8, Ym-11, and Ym-12 deletions, respectively.

For category 4 deletions, patterns 12, 22, 23, 35, 36, 37 and 38 occurred in $5,1,1,1,2,1$, and 1 samples, respectively (Fig. 6). Probe sY1291 is negligible regardless of the absence or presence of band, as described earlier for the Japanese population. Therefore, we classified these deletions as polymorphisms including absence of two deletions.

\section{Discussion}

In Japan, all available genetic testing for AZF deletions is based on the Promega Y Chromosome AZF Analysis System (version
2.0; Promega, Madison, WI, USA). Previously, numerous nonspecific bands were occasionally observed with these PCR products, probably because this system was optimized for Caucasian DNA or different thermocyclers.

In the present study, we evaluated the performance of the GENOSEARCH $^{\mathrm{TM}}$ AZF Deletion kit, which is based on Luminex xMAP technology and identifies samples harboring deletions of sequences represented by STS probes. An automated multiplex bead-array system provided high-throughput identification of PCR products. ${ }^{21}$

Currently, up to 100 spectrally distinct fluorescence-labeled beads are available for multiplex target detection on the Luminex suspension microarray platform. This array system saves labor, money and time, ${ }^{22,23}$ and can be implemented daily with acceptable sensitivities and specificities. Its routine use 


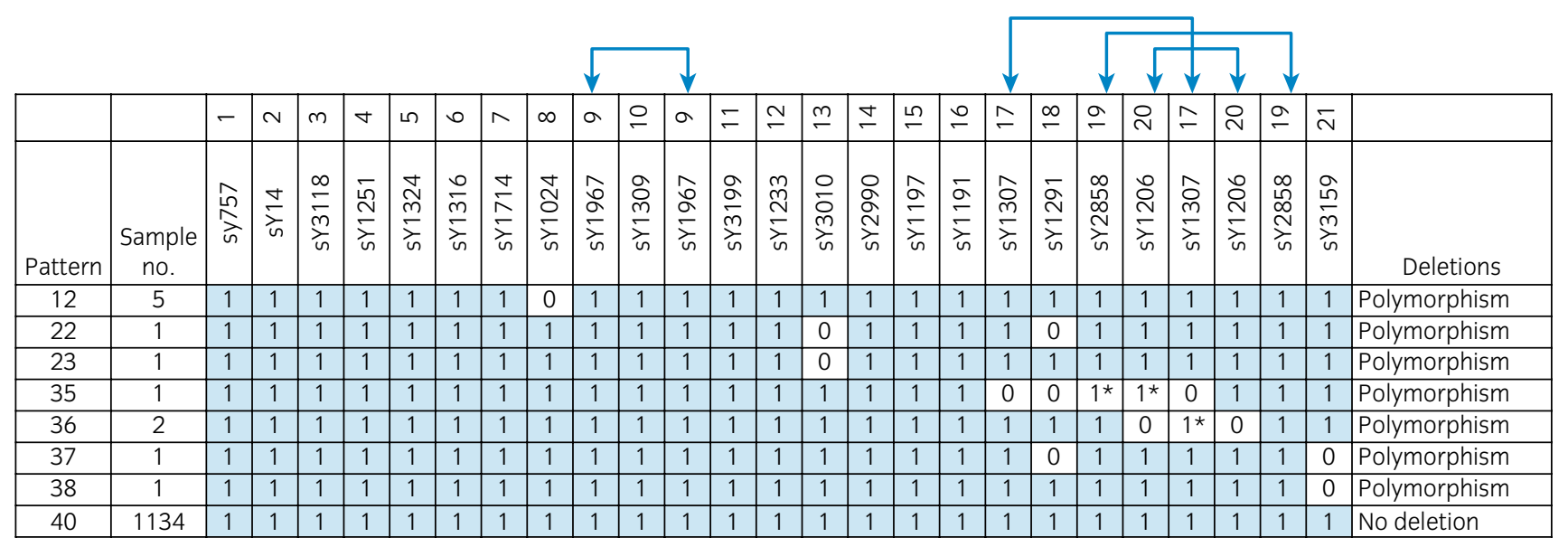

Fig. 6 Miscellaneous deletions (category 4). Blue arrows highlight data from the same probe. Possible deletions are colored light blue.

might underlie the development and implementation of assays to detect genetic deletions in a timely manner.

Our molecular assays were carried out on DNA samples that had undergone several freeze/thaw cycles. Although deletion assays might be improved by further probe optimization, changes in the probe sequences could have deleterious effects on other targets in the multiplexed reaction. Therefore, the GENOSEARCH ${ }^{\mathrm{TM}}$ AZF Deletion kit provides a practical solution in the clinical setting for rapidly screening and detecting AZF deletions.

We detected excellent specificities and favorable sensitivities in comparison with the use of conventional STS probes for the detection of AZF deletions. Furthermore, this kit is compatible with rapid, automated, high-throughput procedures; simultaneous detection strategies reduced the manpower, reagent volumes and specimen volumes required for use of the GENOSEARCH ${ }^{\mathrm{TM}}$ AZF Deletion kit.

From the clinical point of view, microdissection TESE through microscopy is currently recognized as the best way of retrieving sperm in men with non-obstructive azoospermia. Compared with conventional TESE, microdissection TESE has a higher sperm retrieval rate and is safer. ${ }^{24}$ Genetic testing for Y-chromosome microdeletions is of prognostic significance for TESE.

Men with complete deletions of the AZFa or $\mathrm{AZFb}$ region or absence of the $\mathrm{AZFb}+\mathrm{c}$ region have no chance of sperm retrieval during microdissection TESE, ${ }^{25}$ and are not recommended to undergo this procedure. Many unnecessary TESE procedures are carried out, motivating the need for reliable genetic testing.

Category 1 deletions correspond to the AZF deletions referred to by the $2004 \mathrm{EAA} / \mathrm{EMQN}$ guidelines, and are sufficient to make a diagnosis for male infertility. The present results show that the GENOSEARCH ${ }^{\mathrm{TM}}$ AZF Deletion kit might be a suitable replacement for conventional STS probes for Japanese populations. When deletions in category 2 are detected, cytogenetic assays should be carried out for determining the patient's karyotype.

It should be emphasized that microdeletions, excluding the Ym-8, Ym-11 and Ym-12 microdeletions in category 3, are atypical classifications that have not been rigorously investigated. Therefore, the presence of these microdeletions might impact on the interpretation of some clinical findings, although this scenario is expected to rarely occur. The accurate detection of these microdeletions was the most striking feature of the GENOSEARCH $^{\mathrm{TM}}$ AZF Deletion kit.

In conclusion, we have described the development of the GENOSEARCH $^{\mathrm{TM}}$ AZF Deletion kit for the detection of a panel of AZF deletions; this technology includes the use of Luminex XMAP arrays. This new kit provided a routine tool for the diagnosis of AZF deletions in patients accessing a male infertility clinic in Japan. This kit would also be useful for the detection of atypical microdeletions.

\section{Acknowledgments}

Support for this study was provided by the A step Fund (2011) of the Japan Science and Technology Agency (AS231Z01752F)

\section{Conflict of interest}

None declared.

\section{References}

1 Cram DS, O'Bryan MK, de Kretser DM. Male infertility genetics-the future. J. Androl. 2001; 22: 738-46.

2 de Kretser DM, Burger HG. The Y chromosome and spermatogenesis. $N$. Engl. J. Med. 1997; 336: 576-8.

3 Tiepolo L, Zuffardi O. Localization of factors controlling spermatogenesis in the nonfluorescent portion of the human Y chromosome long arm. Hum. Genet. 1976; 34: 119-24.

4 Vogt PH. Genetics of idiopathic male infertility: Y chromosomal azoospermia factors (AZFa, AZFb, AZFc). Baillieres Clin. Obstet. Gynaecol. 1997; 11: 773-95.

5 Skaletsky H, Kuroda-Kawaguchi T, Minx PJ et al. The male-specific region of the human Y chromosome is a mosaic of discrete sequence classes. Nature 2003; 423: 825-37.

6 Kuroda-Kawaguchi T, Skaletsky H, Brown LG et al. The AZFc region of the $\mathrm{Y}$ chromosome features massive palindromes and uniform recurrent deletions in infertile men. Nat. Genet. 2001; 29: 279-86.

7 Ferlin A, Arredi B, Speltra E et al. Molecular and clinical characterization of $\mathrm{Y}$ chromosome microdeletions in infertile men: a 10-year experience in Italy. J. Clin. Endocrinol. Metab. 2007; 92: 762-70. 
8 Katagiri Y, Neri QV, Takeuchi T et al. Y chromosome assessment and its implications for the development of ICSI children. Reprod. Biomed. Online 2004; 8: 307-18

9 Simoni M, Bakker E, Krausz C. EAA/EMQN best practice guidelines for molecular diagnosis of y-chromosomal microdeletions. State of the art 2004 Int. J. Androl. 2004; 27: 240-9.

10 Fukushima M, Koh E, Choi J, Maeda Y, Namiki M, Yoshida A. Reevaluation of azoospermic factor $\mathrm{c}$ microdeletions using sequence-tagged site markers with confirmed physical positions from the GenBank database. Fertil. Steril. 2006; 85: 965-71.

11 Choi J, Koh E, Matsui F et al. Study of azoospermia factor-a deletion caused by homologous recombination between the human endogenous retroviral elements and population-specific alleles in Japanese infertile males. Fertil. Steril. 2008; 89: 1177-82.

12 Y Chromosome Consortium. A nomenclature system for the tree of human Y-chromosomal binary haplogroups. Genome Res. 2002; 12: 339-48.

13 Kuroki Y, Iwamoto T, Lee J et al. Spermatogenic ability is different among males in different Y chromosome lineage. J. Hum. Genet. 1999; 44: 289-92.

14 Hammer MF, Karafet TM, Park H et al. Dual origins of the Japanese: common ground for hunter-gatherer and farmer Y chromosomes. J. Hum. Genet. 2006; 51: 47-58.

15 Krausz C, Giachini C, Xue Y et al. Phenotypic variation within European carriers of the Y-chromosomal gr/gr deletion is independent of Y-chromosomal background. J. Med. Genet. 2009; 46: 21-31.

16 Sin HS, Koh E, Shigehara K et al. Features of constitutive gr/gr deletion in a Japanese population. Hum. Reprod. 2010; 25: 2396-403.

17 Hucklenbroich K, Gromoll J, Heinrich M, Hohoff C, Nieschlag E, Simoni M. Partial deletions in the AZFc region of the $\mathrm{Y}$ chromosome occur in men with impaired as well as normal spermatogenesis. Hum. Reprod. 2005; 20: 191-7.

18 Repping S, Skaletsky H, Brown L et al. Polymorphism for a 1.6-Mb deletion of the human $\mathrm{Y}$ chromosome persists through balance between recurrent mutation and haploid selection. Nat. Genet. 2003; 35: 247-51.
19 Repping S, Korver CM, Oates RD et al. Are sequence family variants useful for identifying deletions in the human Y chromosome? Am. J. Hum. Genet. 2004; 75: 514-17; author reply 517-19.

20 Fernandes S, Paracchini S, Meyer LH, Floridia G, Tyler-Smith C, Vogt PH. A large AZFc deletion removes DAZ3/DAZ4 and nearby genes from men in $\mathrm{Y}$ haplogroup N. Am. J. Hum. Genet. 2004; 74: 180-7.

21 Lalonde MS, Arts EJ. DNA suspension arrays: silencing discrete artifacts for high-sensitivity applications. PLoS One 2010; 5: e15476.

22 Dunbar SA. Applications of Luminex xMAP technology for rapid, high-throughput multiplexed nucleic acid detection. Clin. Chim. Acta 2006; 363: $71-82$.

23 Sun K, Chen XF, Zhu XB et al. A new molecular diagnostic approach to assess y chromosome microdeletions in infertile men. J. Int. Med. Res. 2012; 40: $237-48$.

24 Dabaja AA, Schlegel PN. Microdissection testicular sperm extraction: an update. Asian J. Androl. 2013; 15: 35-9.

25 Schlegel PN. Nonobstructive azoospermia: a revolutionary surgical approach and results. Semin. Reprod. Med. 2009; 27: 165-70.

\section{Supporting information}

Additional Supporting Information may be found in the online version of this article at the publisher's web-site:

Fig. S1 raw deletion data in accordance with various patterns. Within the body of the figure, " 0 " denotes "absence," "1" denotes "presence" and " 1 *" denotes duplicated copies of a DNA sequence. Possible deletions are colored grey. Notation is as in Figure 3.

Table S1 Chromosomal aberrations in category 2 in this study. 